\title{
The New Smart Eraser Design
}

\author{
Jinzan Liu ${ }^{1}$, Zhong Zeng ${ }^{1} \&$ Lang $\mathrm{Xu}^{1}$ \\ ${ }^{1}$ University of Shanghai for Science and Technology, Shanghai, China \\ Correspondence: Jinzan Liu, College of Mechanical Engineering, University of Shanghai for Science and \\ Technology, 516 Jungong Road, Shanghai 200093, China. E-mail: jinzan_Liu@163.com
}

Received: December 5, 2012

Accepted: January 21, $2013 \quad$ Online Published: January 29, 2013

doi:10.5539/mas.v7n2p50

URL: http://dx.doi.org/10.5539/mas.v7n2p50

\begin{abstract}
The paper puts forward a kind of mechanism design scheme, the mechanism can automatically detect the blackboard chalk stains, and erase the font, keep the blackboard clean. In this paper, the hardware system and software have been connected with each other, and tell us how to get installed. It introduces the principle of the mechanism, the method of image processing, and calculates the relationship of the displacement, velocity, acceleration and structure. This wipe mechanism adopts crank slider mechanism which has a good wipe effect.
\end{abstract}

Keywords: blackboard eraser institution, intelligent, wipe, image processing, crank slider mechanism

\section{Introduction}

The traditional blackboard chalk dust is a common problem in the traditional blackboard-eraser-chalk architecture (Ren, 2002). Lots of improved blackboard eraser structure came into being, but these improvements changed the original wipe, or not fundamentally solve the problem, or too costly and difficult to spread (Hu \& Wan, 2008). For this reason, the design is based on the traditional blackboard-eraser-chalk called intelligent wipe chalk system, its connotation of "smart" includes moving, positioning, wipe (Wang, Song, \& Wang, 2009). The design is able to achieve automated clean the blackboard and collect dust, which has a good prospect.

Bodies base on traditional blackboard and eraser size. The mechanism consists of three motors A, B, and C, three guide rails a, b, c, three sliders 1, 2, 3 and a slider-crank mechanism. Slider 1 and slider 4 are connected by rails $\mathrm{c}$, and rails $\mathrm{c}$ is installed on them, can be moved in parallel with the slider, power-driven provided by three motors A, B, C. Motor A drives the left and right movement of beams c, and motor B drives the vertical movement of the slider 3, $\mathrm{C}$ is the power source of the slider-crank mechanism which driven rotary motion slider to rub $\mathrm{c}$ moving up and down along the rail, together with the installation in 2 below the blackboard (Chart 1).

The process is carried out as follows: the power of beam c comes from the motor A, the rail transfers the power. Motor A forward and reverse rotation drives the beam $\mathrm{C}$ forward or back movement. Motor $\mathrm{B}$ forward and reverse rotation drives the slider 3 upward and downward movement. The method is able to reach the special position, and then eraser cleans the blackboard. Motor $\mathrm{C}$ is installed in block 3 internal provides power for Slider-crank mechanism, pushes the slider 2 up-and-down along the rail c. Slider 2 is a combination of the movement of a, b, c, performs wipe. When the wipe is finished, A, B motor work and C motor stop, blackboard eraser returns to the original position, waiting for the next wipe. 


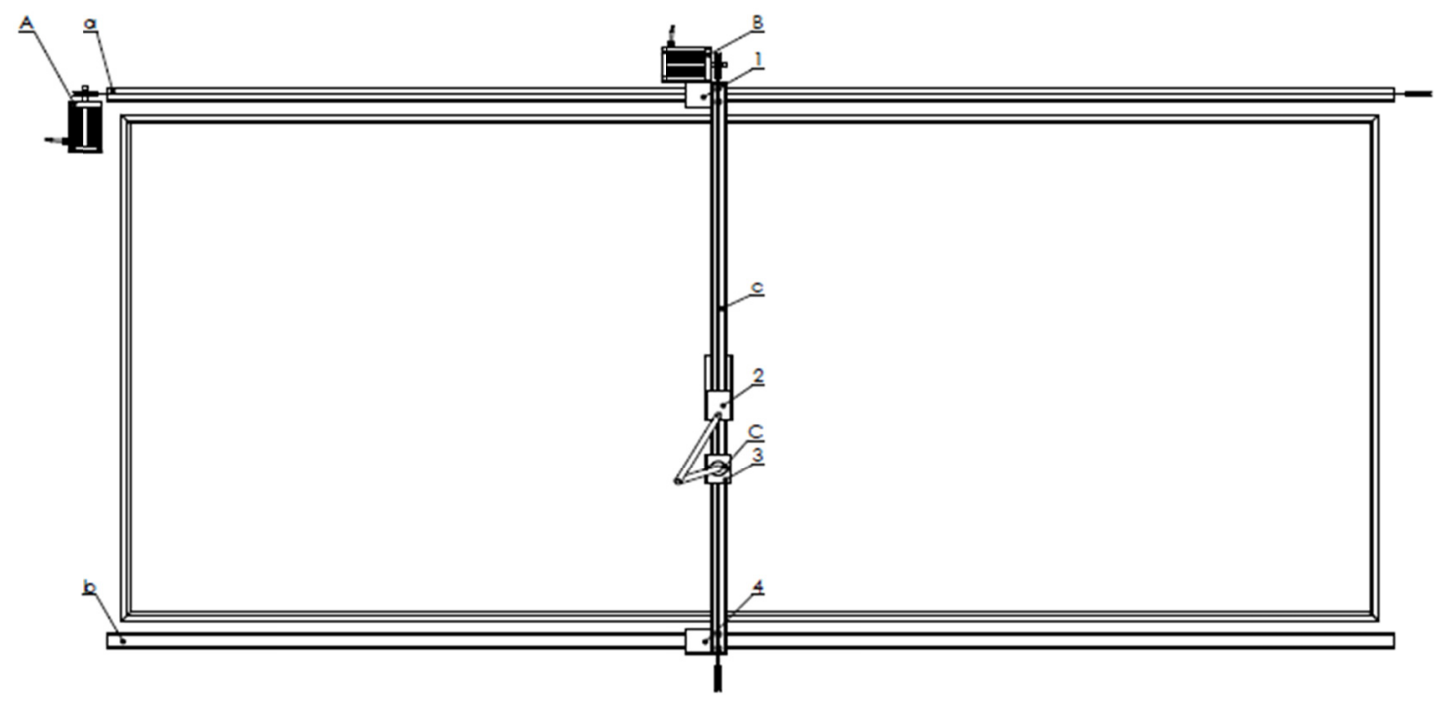

Chart 1. Mechanical structure

The blackboard surface is as the X-O-Y plane.

When the eraser begins to wipe, beam is at the left-most of the blackboard, and eraser on the top. Beam and eraser back to the original position after wiping completed. Before the eraser to wipe, CCD camera takes pictures of the entire blackboard. In the program, the stains are contained in the rectangle. By computer processing, the program determine the coordinates of the stains, PC can calculate the upper left corner of the rectangle's coordinate, this is the coordinate which the eraser should be reached. Then the motors release pulse signal, motors rotate a certain number of turns, eraser arrives at the designated location (Chart 2).

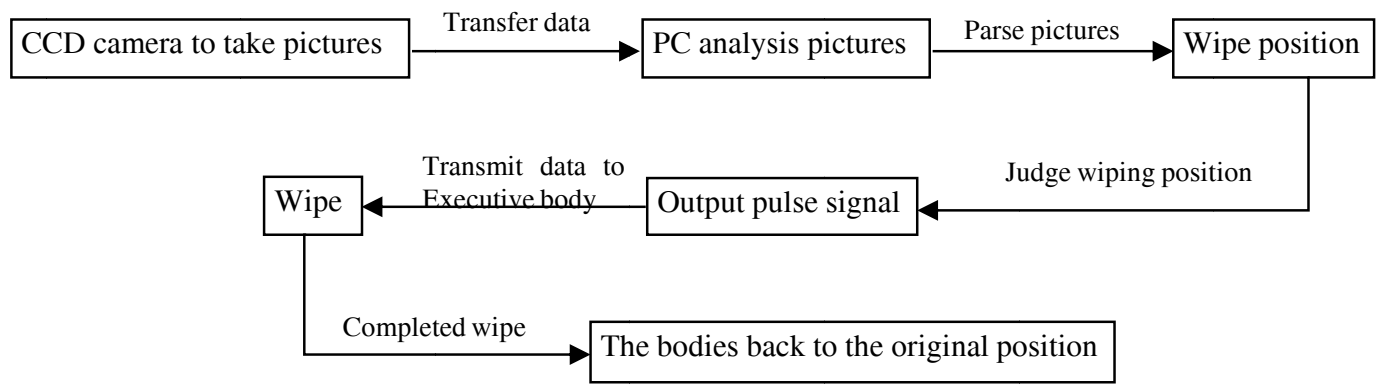

Chart 2. Flow chart

\section{Visual Acquisition and Control}

The automatic blackboard wipe system is divided into two parts of the information processing unit and motion control unit, whose hardware selection is run around the two parts (Zhang, 2008). The hardware of information processing unit involves to lighting source, CCD camera, lens, scene collect card \& connection line parts and so on, whose main task is to use the image in the CCD camera collecting from the blackboard, then for analysis and processing of the acquired images to obtain the info. The blackboard is different from the background color of the blackboard surface. Finally output this information to provide the coordinates for the eraser wiping execution reference (Qian, 2005). The motion control unit includes motion control card, servo drives, servo motors and sensors, whose main task is to control the speed of the slider by the servo motor, make the eraser finish the wiping action successful (Yang \& Chong, 2007).

The hardware of information processing (Deng, 2002; Zhou \& Wu, 2008) unit involved mainly CCD cameras, lenses, frame grabbers and the connection line accessories. Motion control unit related to the hardware mainly have motion control card, servo drives, servo motors and sensors. The agency will choose the PC system as the hardware development platform, the visual system will choose the PCI interface frame grabber and CCD image sensors, motion control system will choose the PCI interface motion control card and servo motion controller. 
The control system software design requires software platform with easy-to-use, modular, standardize and repeatable development features. Choosing a graphical programming language LabView make it convenient for maintenance and improvement.

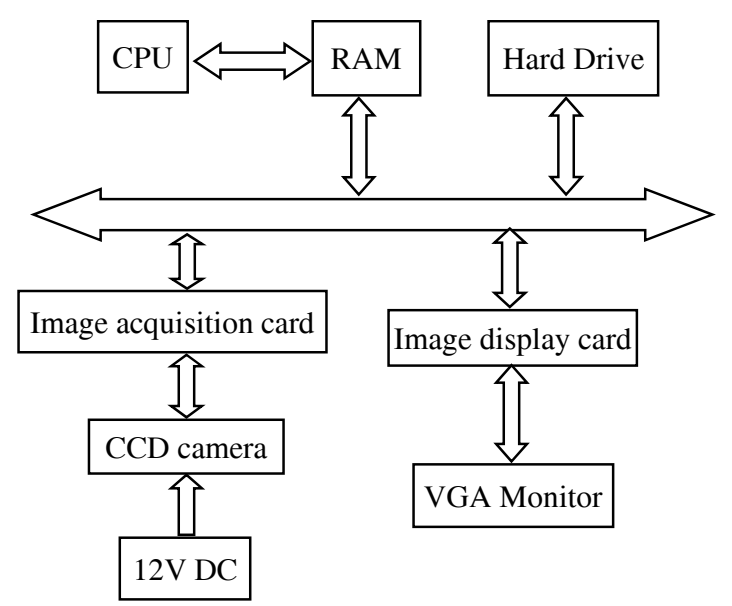

Figure 1. The acquisition system

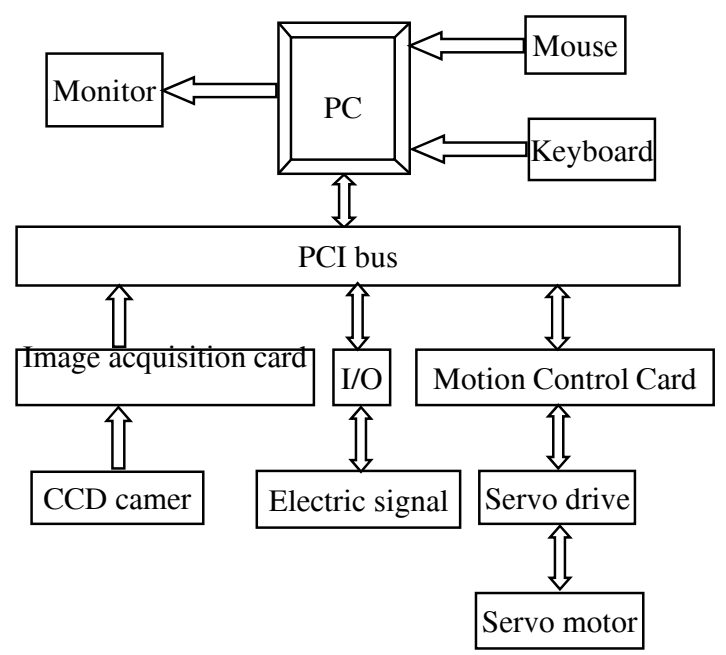

Figure 2. Control System Design

\section{Image processing}

Image acquisition is a very important step in the visual collection. Because the unevenness of the CCD sensitivity and quantizing noise in photovoltaic conversion process is existent, and the reason of light. Actually, the quality of the image becomes poor, we cannot get a clear image. In order to facilitate the subsequent image analysis and understanding, it is necessary for image processing.

(1) Image pretreatment

Image pretreatment contains image smoothing (Grosky \& Tamburino, 1990; Sbnchez \& Martinez, 2000; Sun \& $\mathrm{Fu}, 2005)$ and image sharpening (Cai, Peng, \& He, 2003).

The purpose of image smoothing is to suppress the image noise and improve image quality. In the practical application, the image noise source is come from blackboard surface's spots and the thermal noise of the flow of electron. When the noise reaches a certain intensity, will make subsequent image processing work cannot be carried out, so the image processing smoothing is required. In order to get a better picture of the edge information, the next is image sharpening. The purpose of sharpening processing of the image is mainly used to enhance the outline of the edge in the image, details and gradation to form a complete boundary of the object, to achieve the separation of the object from the image, or indicating that the surface region of the same object is detected. The Gradient and Laplace operator due to the isotropic nature, and therefore is a commonly used 
method for sharpening. Gradient method is direct, simple, fast operation, but where there is a big change of image grayscale, there is a gradient's image output, but homogeneous regions in grayscale-scale gradient value is zero, so this method only display the edge contour where there is a big change of image grayscale.

Laplace is also a common edge enhancement processing operator, to enhance boundary contour. But it should be noted that, while using the Laplace operator sharpen the image, the image noise will be came together. Then enhance the image edge will enhance the image noise, the Laplace image sharpening first image smoothing. Before Laplace image sharpening, the first is image smoothing. In summary, Laplace as the subject sharpening method is suitable for selection under the premise of the smoothed image, the method can enhance the image edge, anti-interference, high positioning accuracy border and the edge of the continuous is good.

$$
\text { Image segmentation }
$$

Image segmentation is based on some of the characteristics of the image similarity criteria, grouping the image pixel, the image plane is divided into a series of special area, it will reduce the data which should be processed by the stage of the image analysis, recognition, etc. while retaining the information about the image structure characteristics. The paper uses threshold method to achieve image segmentation algorithm. The threshold is the method which we put each pixel to compare with the threshold value $T$, and we determined its category according to whether the grayscale value exceeds the threshold value. In this paper, the peak-to-valley method is determined segmentation threshold. The principle is: If the target area and the background area have some differences in grayscale image, then the histogram of image grayscale is bimodal shape, that is to say, the target and background pixel according to its probability in the image-grayscale histogram will form the respective crest and a trough between the two peaks occurs, so choose the pixel grayscale scale value of the trough at the image segmentation can be used as the threshold value $\mathrm{T}$ (chart 3). In this paper, chalk color and background color have a lot of contrast, so we can get a better bimodal pattern.

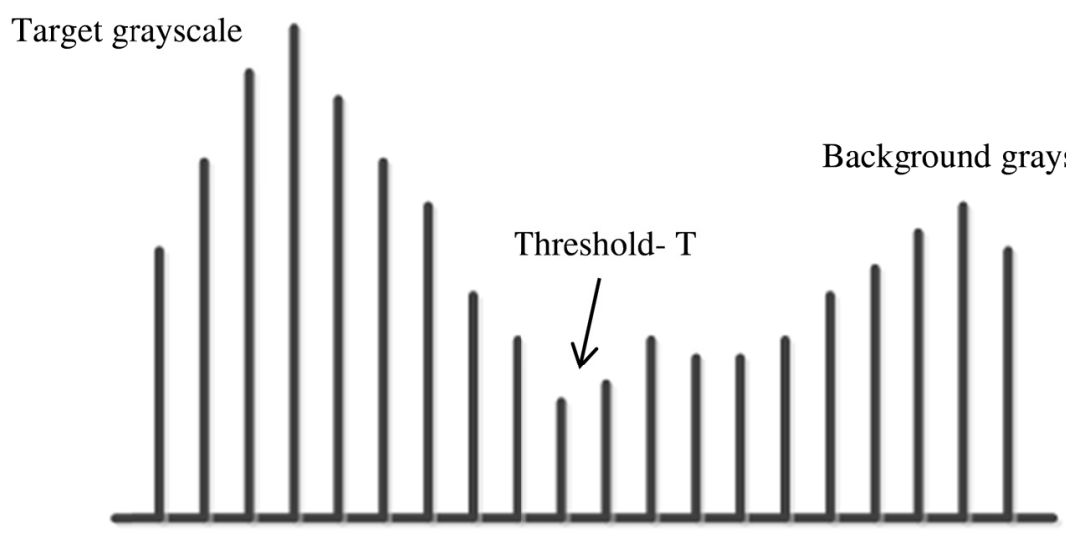

Chart 3. Image Grayscale

\section{Slider-crank Mechanism Motion Analysis (Pu \& Ji, 2001)}

First, establishing a Cartesian coordinate and marking the vector and the azimuth of the rods. Known: the eraser width of $\mathrm{D}$, A motor drives beam $\mathrm{c}$, speed is $\mathrm{V}_{\mathrm{x}}$, the motor $\mathrm{C}$ drives slider-crank perform wiping action.

$$
\begin{gathered}
\mathrm{V}_{\mathrm{cy}}=\mathrm{L}_{1} \cdot \cos \theta \\
\mathrm{L}_{3}=\mathrm{L}_{1} \cdot \sin \theta+\mathrm{L}_{2} \cdot \cos \beta \\
\mathrm{l}_{2} \cdot \sin \beta=\mathrm{l}_{1} \cdot \cos \theta
\end{gathered}
$$

(1) The first derivative with respect to time:

$$
\alpha_{\mathrm{cy}}=-\mathrm{L}_{1} \cdot \omega \sin \omega \mathrm{t}
$$

Blackboard eraser in the midline wipes to repeat $\mathrm{D} / 2$ traces, when the eraser is horizontal movement $\mathrm{D} / 2, \boldsymbol{L}_{1}$ rotational is half cycle: 


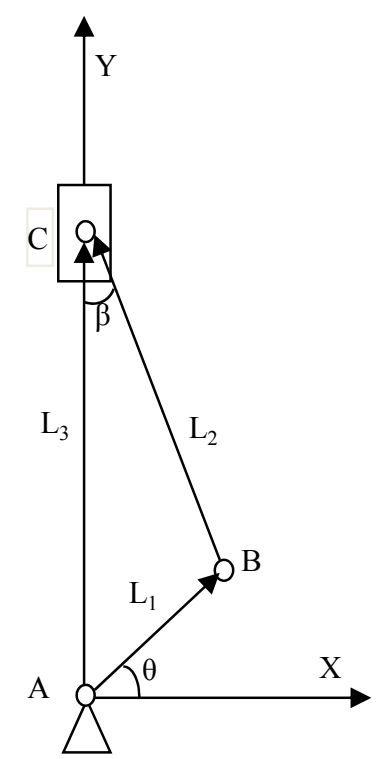

$$
\begin{gathered}
\frac{D}{2 v_{t}}=\frac{T}{2} \\
\omega=\frac{2 \pi}{T}
\end{gathered}
$$

$$
\mathrm{L}_{3}=\mathrm{L}_{1} \cdot \sin \theta+\cos \theta \sqrt{\mathrm{L}_{1}^{2}-\mathrm{L}_{2}^{2}}
$$

We can get the conclusion $\mathrm{v}_{\mathrm{c}}, \alpha_{\mathrm{c}}, \mathrm{L}_{\mathrm{c}}$ :

$$
\begin{array}{r}
\mathrm{V}_{\mathrm{c}}=\mathrm{V}_{\mathrm{x}}+\mathrm{L}_{1} \cdot \cos \omega \mathrm{t} \\
\mathrm{v}_{\mathrm{c}}=\sqrt{\mathrm{v}_{\mathrm{x}}^{2}+\mathrm{v}_{\mathrm{cy}}^{2}} \\
=\sqrt{\mathrm{v}_{\mathrm{x}}^{2}+\left(\mathrm{L}_{1} \cdot \cos \omega \mathrm{t}\right)^{2}} \\
\alpha_{\mathrm{cy}}=\alpha_{\mathrm{cy}} \\
=-\mathrm{L}_{1} \cdot \omega \sin \omega \mathrm{t} \\
\mathbf{S}_{\mathrm{c}}=\mathbf{L}_{1} \sin \omega \mathrm{t}+\mathrm{V}_{\mathrm{x}} \mathrm{t}+\cos \omega \mathrm{t} \sqrt{\mathbf{L}_{1}^{2}-\mathbf{L}_{2}^{2}}
\end{array}
$$

Written in the form of a matrix:

$$
\left(\begin{array}{ccc}
0 & \mathrm{~L}_{1} & \mathrm{~V}_{\mathrm{x}} \\
-\mathrm{L}_{1} \omega & 0 & 0 \\
\mathrm{~L}_{1} & \sqrt{\mathrm{L}_{1}^{2}-\mathrm{L}_{2}^{2}} & \mathrm{~V}_{\mathrm{x}} \mathrm{t}
\end{array}\right)\left(\begin{array}{c}
\sin \omega \mathrm{t} \\
\cos \omega \mathrm{t} \\
1
\end{array}\right)=\left(\begin{array}{c}
\mathrm{V}_{\mathrm{x}} \\
\alpha_{c} \\
\mathrm{~S}_{\mathrm{c}}
\end{array}\right)
$$

Already know D=60 mm, $\boldsymbol{L}_{1}=100 \mathrm{~mm}, \boldsymbol{L}_{2}=180 \mathrm{~mm}, \boldsymbol{V}_{\mathrm{x}}=6 \mathrm{~mm} / \mathrm{s}$, we can draw the graphics: 


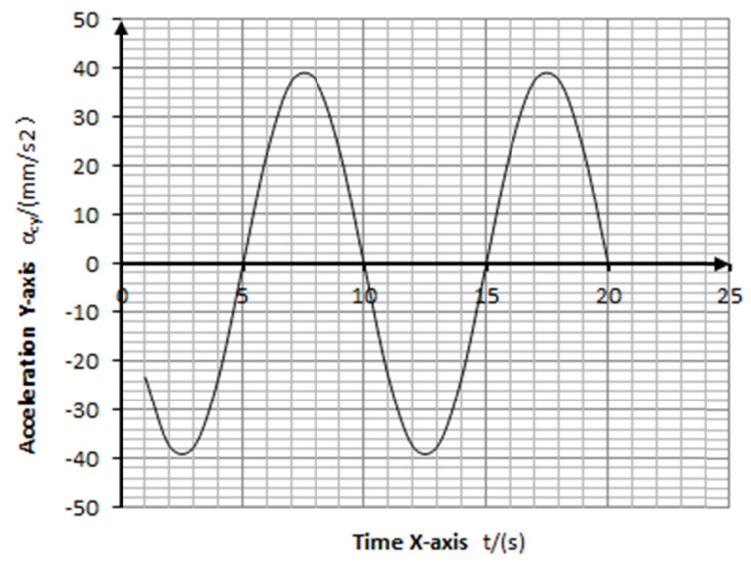

- $\alpha c y-T$ The point $\mathrm{C}$ on the $\mathrm{Y}$-axis direction acceleration

Figure 3. Acceleration Y-axis

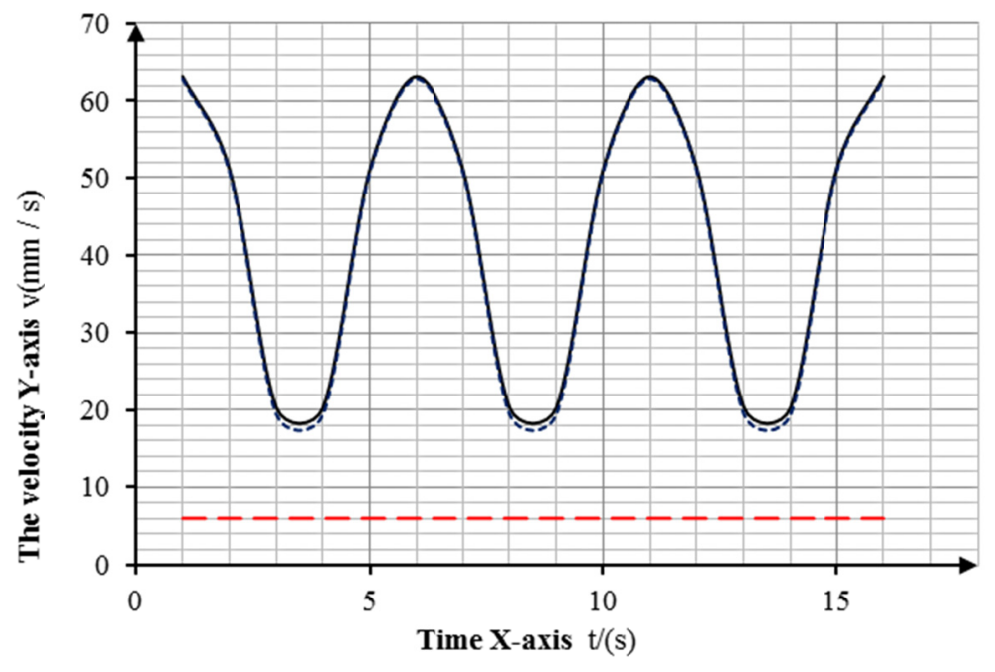

- Vc-The speed of the point C

- - $-\mathrm{Vx}-\mathrm{The}$ point $\mathrm{C}$ on the $\mathrm{X}$-axis direction speed

----- Vcy-The point $\mathrm{C}$ on the $\mathrm{Y}$-axis direction speed

Figure 4. The velocity $\mathrm{Y}$-axis

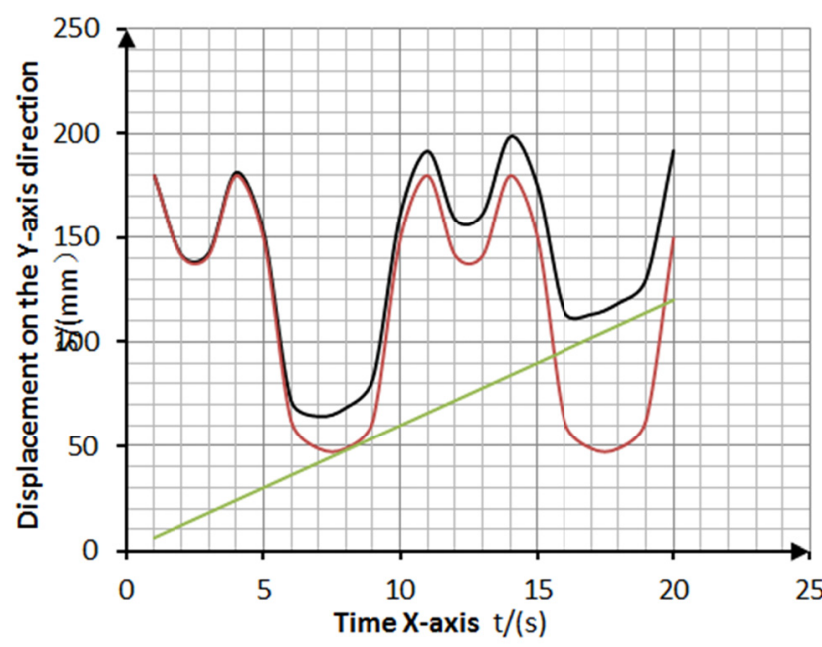

- S-The displacement of the point C

- Sy-The point $\mathrm{C}$ on the $\mathrm{Y}$ axis direction displacement

- Sx-The point $C$ on the $X$ axis direction displacement

Figure 5. Displacement on the Y-axis direction 
According to the calculation and analysis, we get a conclusion that blackboard erase is able to run smooth, and complete the expected movement.

\section{Summary}

Compared with manually wipe, smart wipe has a good effect and runs smooth with good reaction speed. The rate of rotation of the motor can be set in accordance with the requirements of the wiping speed to suit the requirements of different occasions.

1) The smart eraser has a simple structure, easy to operate, easy to obtain raw materials, manufacturing equipment simple process.

2) Control functions, and less susceptible to interference, high reliability, ease of use, can make products with high performance and low cost.

3) The product is suitable for large, medium and small institutions, the promotion of a certain significance.

\section{References}

Cai, Y. J., Peng, T., \& He, J. (2003). The Research of Packaging-Checking Based on Application of Machine Vision. Packaging Engineering, 24(2), 45-48.

Deng, X. Z. (2002). Electric Drive Control. Wuhan: Hua zhong University of Science and Technology Press.

Grosky, W. I., \& Tamburino, L. A. (1990). A Unified Approach to the Linear Camera Calibration Problem. IEEE Transactions on Pattern Analysis and Machine Intelligence, 12(7), 663-671. http://dx.doi.org/10.1109/34.56209

Hu, Y. K., \& Jing, R. C. (2006). Theoretical Mechanics. Beijing: Higher Education Press.

Hu, Z. Y., \& Wan, H. (2006). A pressure cleaner of blackboard eraser. Journal of Wuhan Institute of Shipbuilding Technology, Wuhan.

Pu, L. G., \& Ji, M. G. (2001). Mechanical design (7th ed.). pp. 167-170. Beijing: Higher Education Press.

Qian, H. (2005). Key Technology of the Vision System of High-speed Parallel Robot. Tianjin: Tianjin University.

Ren, Z. G. (2002). Chalk dust on the health hazards of teachers (in Chinese). Chinese Journal of School Health, 2, 189.

Sbnchez, A. J., \& Martinez, J. M. (2000). Robot-arm pick and place behavior programming system using visual perception. Proceedings 15th International Conference on Pattern Recognition, 4, 507-510.

Sun, H. Y., \& Fu, D. S. (2005). Multi-target tracking technology in the video surveillance system. Computer Applications and Software, 22(11), 22-25.

Wang, X. F., Song, X. T., \& Wang, Z. (2009). Innovative Design of a New Mechanism to Clean the Blackboard. Mechanical Engineer, 10.

Yang, S. Z., \& Chong, Y. K. (2007). Mechanical engineering control. Wuhan: Hua zhong University of Science and Technology Press.

Zhang, C. (2008). $R \& D$ of Vision Control System for a High Speed Packaging Robot. Tianjin: Tianjin University.

Zhou, H. Q., \& Wu, Q. X. (2008). Microcomputer Principle and Interface Technology (4th ed.). Hefei: University of Science and Technology of China Press. 